\title{
NASA LK-172,140
}

\section{NASA Contractor Report 172140}

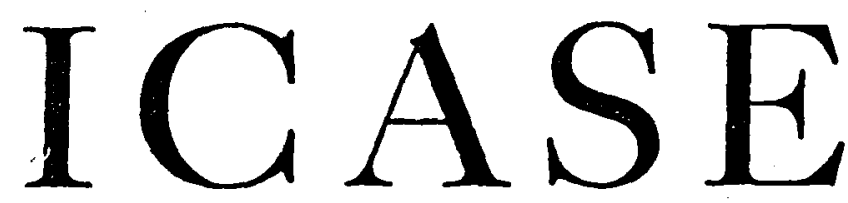

ON THE CONTROL CANONICAL STRUCTURE OF A CLASS OF SCAIAR INPUT SYSTEMS

Russe11 Teglas

Contract No. NAS1-15810

June 1983

INSTITUTE FOR COMPUTER APPLICATIONS IN SCIENCE AND ENGINEERING NASA Langley Research Center, Hampton, Virginia 23665

Operated by the Universities Space Research Association

\section{LIBRARY EOPY}

\section{N/Sก \\ National Aeronautics and Space Administration

.ง! 111983

LANGLEY RESEARCH CENTER LIBRARY, NASA HAMPTON, VIRGINIA

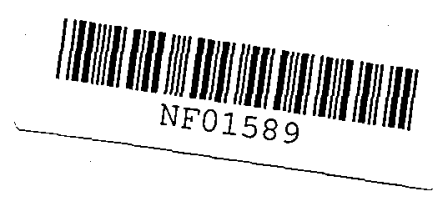


ON THE CONTROL CANONICAL STRUCTURE OF A

CLASS OF SCALAR INPUT SYSTEMS

\author{
Russell G. Teglas \\ Institute for Computer Applications in Science and Engineering
}

\title{
ABSTRACT
}

In this paper, we demonstrate the equivalence of a scalar input system $\dot{x}=\mathscr{A} x+b u$, for which the eigenvalues of the generator $\mathscr{A}$ colncide with the roots of the entire function

$$
p(\omega)=e^{\omega T}+a_{1} e^{\omega\left(T-\theta_{1}\right)}+\cdots+a_{m}+\int_{0}^{T} \cdot a(\theta) e^{\omega(T-\theta)} d \theta
$$

with the controlled scalar functional equation

$$
y(t)+a_{1} y\left(t-\theta_{1}\right)+\cdots+a_{m} y(t-T)+\int_{0}^{T} a(\theta) y(t-\theta) d \theta=u(t)
$$

The theory of nonharmonic Fourier series is then employed to investigate the placement of eigenvalues in the closed-loop system with continuous state feedback.

This research was sponsored in part by the U. S. Air Force office of Scientific Research under Grant No. AFOSR-79-0018 and in part by the National Aeronautics and Space Administration under NASA Contract No. NAS1-15810 while the author was in residence at ICASE, NASA Langley Research Center, Hampton, VA 23665 . 


\section{Introduction}

Let $\mathscr{A}$ be a generator of a strongly continuous group of operators $\{\mathscr{G}(t): t \in R\}$ on a Hilbert space $\mathscr{E}$, and suppose that the spectrum of $\mathscr{A}$ consists of an infinite sequence of simple eigenvalues $\left\{w_{k}\right\}$ which forms the zero set of an entire function having the form

$$
p(\omega)=e^{\omega T}+a_{1} e^{\omega\left(T-\theta_{1}\right)}+\cdots a_{m}+\int_{0}^{T} a(\theta) e^{\omega(T-\theta)} d \theta .
$$

In this paper, we will analyze in detail the transformation which carries the scalar input system

$$
x(t)=\mathscr{A} x(t)+b u(t)
$$

to the scalar functional equation

$$
y(t)+a_{1} y\left(t-\theta_{1}\right)+\cdots+a_{m} y(t-T)+\int_{0}^{T} a(\theta) y(t-\theta) d \theta=u(t)
$$

The latter constitutes the causal control canonical form for the pair $(\mathscr{A}, b)$. The adjective "causal" is used here because a feedback law of the form

$$
u(t)=(6, x(t))_{\mathscr{E}}
$$

wi11 be shown to transform to a feedback law of the form

$$
u(t)=\int_{0}^{T} g(\theta) y(t-\theta) d \theta
$$

1.e., the input $u$ depends only upon past values of $y$. The expression 
"control; canonical form" refers to the fact that the above-mentioned transformation, has a structure quite similar to its well-known finite dimensional counterpart as well as to the fact that the effect of linear feedback (4) in the orfginal system (2) can be readily analyzed in terms of the system (3) with corresponding feedback (5).

Before proceeding, we present an example of a system which possesses the structure indicated above. Consider the linear hyperbolic system in characteristic normal form [3]:

$$
\frac{\partial}{\partial t}\left[\begin{array}{c}
\mathrm{w}^{-} \\
\mathrm{w}^{+}
\end{array}\right]=\left[\begin{array}{cc}
\Lambda^{-} & 0 \\
0 & \Lambda^{+}
\end{array}\right] \frac{\partial}{\partial \mathrm{x}}\left[\begin{array}{c}
\mathrm{w}^{-} \\
\mathrm{w}^{+}
\end{array}\right]+\mathrm{A}(\mathrm{x})\left[\begin{array}{c}
\mathrm{w}^{-} \\
\mathrm{w}^{+}
\end{array}\right],
$$

with boundary conditions

$$
\mathrm{w}^{-}(0, t)=\mathrm{D}_{0^{-}} \mathrm{w}^{+}(0, t) \quad ; \quad \mathrm{w}^{+}(1, t)=\mathrm{D}_{1^{-}}(1, t) .
$$

Here,

$$
\begin{aligned}
& \Lambda^{-}(x)=\operatorname{diag}\left(\lambda_{1}(x), \cdots, \lambda_{n}(x)\right), \\
& \Lambda^{+}(x)=\operatorname{diag}\left(\lambda_{n+1}(x), \cdots, \lambda_{2 n}(x)\right),
\end{aligned}
$$

are diagonal $n \times n$ matrices with

$$
\lambda_{1}(x)<\cdots \leqslant \lambda_{n}(x)<0<\lambda_{n+1}(x)<\cdots \leqslant \lambda_{2 n}(x) .
$$

Also,

$$
A(x)=\left[\begin{array}{ll}
A^{-}(x) & A_{12}(x) \\
A_{21}(x) & A^{+}(x)
\end{array}\right] \text {, }
$$


and $D_{0}$ and $D_{1}$ are both $n \times n$ matrices which determine the manner in which "Information" is reflected at the boundaries $x=0$ and $x=1$. Such systems arise in the study of counterflow heat exchangers [9] and in the study of multiconductor transmission lines [6].

If $A_{12} \equiv A_{21} \equiv 0$ and the off-diagonal components of both $A^{-}$and $A^{+}$ vanish identically, then the characteristic frequencies are the roots of

$$
\pi(\omega)=\operatorname{det}\left[\exp \left(\omega \Lambda_{+}+M_{+}\right)-D_{1} \exp \left(\omega \Lambda_{-}+M_{-}\right) D_{0}\right]
$$

where

$$
\Lambda_{ \pm} \equiv \int_{0}^{1}\left(\Lambda^{ \pm}(\mathrm{x})^{-1} \mathrm{dx},\right.
$$

and

$$
M_{ \pm} \equiv \int_{0}^{1}\left(\Lambda_{ \pm}(x)^{-1} A^{ \pm}(x) d x\right.
$$

The roots of $\pi$ will coincide with those of a function $p$ having the form displayed in (1):

$$
\begin{aligned}
\pi(\omega) & =e^{\omega \alpha}\left(e^{\omega T}+a_{1} e^{\omega\left(T-\theta_{1}\right)}+\cdots+a_{m}\right) \\
& \equiv e^{\omega \alpha} \cdot p(\omega) .
\end{aligned}
$$

In the case where $A$ has non-zero off-diagonal components, one can show [19], under the assumption that both $D_{0}$ and $D_{1}$ are invertible, that $p$ remains the same except for the addition of an integral term as in (1) with $a(\cdot) \varepsilon L^{2}(0, T)$.

In the above example, one may consider control problems wherein one introduces a forcing term proportional to a scalar function $u(t)$ in the 
differential equations (distributed control):

$$
\frac{\partial w}{\partial t}=\Lambda(x) \frac{\partial w}{\partial x}+A(x) w+b(x) u(t)
$$

or in the boundary conditions (point control): e.g.,

$$
\mathrm{w}^{+}(1, \mathrm{~T})=\mathrm{D}_{1} \mathrm{w}^{-}(1, \mathrm{~T})+\mathrm{bu}(\mathrm{t})
$$

Either way, one is led to consider a system of the form

$$
\dot{x}(t)=\mathscr{A} x(t)+b u(t),
$$

where the element $b$, in the former case, lies in the state space $\&$ but, in the latter case, must be interpreted as a distribution. We will discuss this point in greater detail in Section 2.

of central interest in this paper is the spectral synthesis problem: given a set of "desired" eigenvalues $\left\{v_{k}\right\}$, can one construct a feedback law of the form (4) such that the eigenvalues of the closed-loop system

$$
\dot{x}=\operatorname{edx}+b(f, x) \equiv(\mathscr{t}+b \quad \otimes f) x,
$$

colncide precisely with $\left\{v_{k}\right\}$ ? Russell [14] has carried out a study of this question in the case corresponding to $m=1$ above for a class of linear hyperbolic systems consisting of a pair of equations. In his study, the theory of nonharmonic Fourier series is used to study the canonical equation (3) and conclude that any sequence $\left\{v_{k}\right\}$ for which 


$$
\left\{\left(\omega_{k}-v_{k}\right) / b_{k}\right\} \varepsilon \ell^{2}
$$

where $\left\{b_{k}\right\}$ is the sequence of expansion coefficients of the control distribution element $b$ with respect to the efgenfunctions of $\mathscr{A}$, may be synthesized by continuous linear state feedback. An alternative approach, which apparently avoids an appeal to the theory of nonharmonic Fourier series, has been offered by Clarke and Williamson [2]. Perturbation theorems for spectral operators have been employed by Sun [18] to show that condition (8) is both necessary and sufficient for a class of systems that would include the one studied in this paper. The main contributions of this paper include a more explicit representation of the transformation carrying (2) into (3) than that given in [14] as well as a formula for the expansion coefficients of in terms of the desired set of eigenvalues $\left\{v_{k}\right\}$.

\section{A Discrete Finite Dimensional System}

For the sake of motivation, we will review briefly the reduction of the finite dimensional discrete system, where $A \varepsilon \mathbf{R}^{\mathrm{n} \times \mathrm{n}}$ and $\mathrm{b} \varepsilon \mathbf{R}^{\mathrm{n}}$,

$$
x_{k}=A x_{k-1}+b u_{k}
$$

to its causal canonical scalar equation

$$
y_{k}+a_{1} y_{k-1}+\cdots+a_{n} y_{k-n}=u_{k}
$$

where $\operatorname{det}(\lambda I-A) \equiv \lambda^{n}+a_{1} \lambda^{n-1}+\cdots+a_{n} \cdot$ 
For $r=1, \cdots, n$, we have :

$$
x_{k+r}=A^{r} x_{k}+\sum_{\ell=1}^{r} A^{r-\ell} b u_{k+\ell}
$$

In particular, if $x_{0}=0$,

$$
x_{n}=\left[\begin{array}{llll}
A^{n-1} & b & \cdots b & b
\end{array}\right]\left[\begin{array}{c}
u_{1} \\
\vdots \\
u_{n}
\end{array}\right] \equiv C u \text {. }
$$

Well known is the fact that the pair $(A, b)$ is controllable just in case the $\mathrm{n} \times \mathrm{n}$ matrix $\mathrm{C}$ is invertible.

With $a_{0} \equiv 1$, we find

$$
\begin{aligned}
x_{k+n}+a_{1} x_{k+n-1}+\cdots+a_{n} x_{k}=\sum_{m=0}^{n-1} a_{m} x_{k+n-m}+a_{n} x_{k} \\
=\sum_{m=0}^{n-1} a_{m}\left(A^{n-m} x_{k}+\sum_{\ell=1}^{n-m} A^{n-m-l} b u_{k+l}\right)+a_{n} x_{k} \\
=p(A) x_{k}+\sum_{\ell=1}^{n} \sum_{m=0}^{n-l} a_{m} A^{n-m-l} b u_{k+l},
\end{aligned}
$$

where $p(A)=A^{n}+a_{1} A^{n-1}+\cdots+a_{n} I=0$ by the Cayley-Hamilton theorem and where we have interchanged the order of summation in obtaining the last line. The remaining double sum may be rewritten as

$$
\sum_{\ell=1}^{n} \sum_{m=0}^{n-\ell} a_{m} A^{n-m-\ell} b u_{k+\ell}=C M\left[\begin{array}{c}
u_{k+1} \\
\vdots \\
u_{k+n}
\end{array}\right],
$$

where 


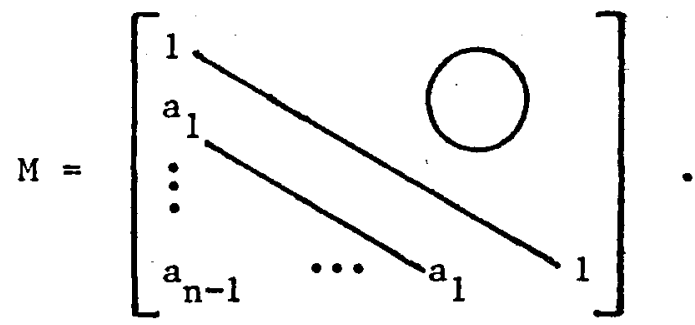

Equation (1.5) now reads

$$
x_{k+n}+a_{1} x_{k+n-1}+\cdots+a_{n} x_{k}=C M\left[\begin{array}{c}
u_{k+1} \\
\vdots \\
u_{k+n}
\end{array}\right] .
$$

It is important to note that the coefficients appearing on the left hand side of (1.7) are scalars. Assuming the pair $(\mathrm{A}, \mathrm{b})$ to be controllable, or equivalently, the matrix $C$ to be invertible, we define the variable $\eta$ by

$$
\left[\begin{array}{c}
\eta_{k, 1} \\
\vdots \\
\eta_{k, n}
\end{array}\right]=M^{-1} C^{-1} x_{k+n}
$$

It is also important that $\eta_{k, r}$ depends only on $k+r$. This may be demonstrated as follows. Let $e_{r}$ denote the $r^{\text {th }}$ standard unit vector in $\mathbf{R}^{\mathrm{n}}$; thus

$$
n_{k, r}=e_{r}^{T_{M}-1} c^{-1} x_{k+n}
$$

According to $(1.8)$,

$$
\left[\begin{array}{c}
\eta_{k+p, 1} \\
\vdots \\
\eta_{k+p, n}
\end{array}\right]=M^{-1} C^{-1} x_{k+p+n}
$$


Suppose $\mathrm{ptq}=\mathrm{r}$. We wish to show that

$$
\eta_{k, r}=\eta_{k+p, q}
$$

1.e, that

$$
\mathrm{e}_{\mathrm{r}^{\mathrm{T}}}^{\mathrm{T}}{ }^{-1} \mathrm{C}^{-1} \mathrm{x}_{k+\mathrm{n}}=\mathrm{e}_{\mathrm{q}^{\mathrm{M}}}^{\mathrm{T}} \mathrm{C}^{-1} \mathrm{x}_{k+p+n}
$$

Since

$$
x_{k+p+n}=A^{P} x_{k+n}+\sum_{\ell=1}^{P A} A^{p-\ell} b u_{k+\ell+n},
$$

we must have:

$$
\begin{aligned}
& \text { (i) } \quad \mathrm{e}_{\mathrm{r}}^{\mathrm{T}}=\mathrm{e}_{\mathrm{q}}^{\mathrm{T}} \mathrm{M}^{-1} \mathrm{C}^{-1}{ }_{\mathrm{A}}^{\mathrm{p}} \mathrm{CM} \equiv \mathrm{e}_{\mathrm{q}}^{\mathrm{T}} \hat{A}^{\mathrm{p}}
\end{aligned}
$$

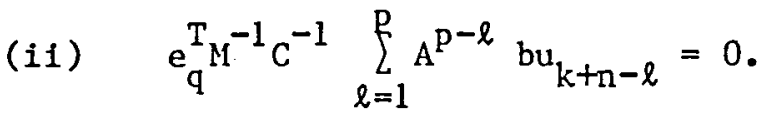

That (i) is true follows from the we11-known fact (see [10] or [16], e.g.) that $\hat{A}$ is the companion matrix of. A:

$$
\hat{A}=M^{-1} C^{-1} A C M=\left[\begin{array}{ccccc}
0 & 1 & & 0 \\
0 & 0 & 1 & 0 \\
0 & 0 & \ldots & 0 & 1 \\
0 & 0 & -a_{2} & -a_{1}
\end{array}\right] .
$$

That (ii) holds true follows from the observation that

$$
\mathrm{C}^{-1} \mathrm{~A}^{\mathrm{p}-\ell} \mathrm{b}=\mathrm{e}_{\mathrm{n}-\mathrm{p}+\ell}
$$

and the fact that $\operatorname{span}\left\{\mathrm{e}_{n-p+\ell}, \cdots, e_{n}\right\}$ is invariant under $M^{-1}$. 
We now define the sequence $\left\{y_{k}\right\} \mathbf{R}$ by

$$
\mathrm{y}_{\mathrm{k}+1}=\eta_{\mathrm{k}, 1}
$$

Equation (1.8) now reads

$$
\left[\begin{array}{c}
y_{k+1} \\
\vdots \\
y_{k+n}
\end{array}\right]=M^{-1} C^{-1} x_{k+n}
$$

Nultiplying (1.7) on the left by $\mathrm{M}^{-1} \mathrm{C}^{-1}$, we find that $\left\{\mathrm{y}_{\mathrm{k}}\right\}$ satisfies (1.2). Let us now see how linear feedback in (1.1) manifests itself in (1.2). If

$$
\mathrm{u}_{\mathrm{k}}=\mathrm{f}^{\mathrm{T}} \mathrm{x}_{\mathrm{k}-1}, \quad \mathrm{f}^{\mathrm{T}}=\text { row vector }
$$

then, by 1.8 ,

$$
u_{k}=f^{T} C M\left[\begin{array}{c}
y_{k-n} \\
\vdots \\
y_{k-1}
\end{array}\right] \equiv g^{T}\left[\begin{array}{c}
y_{k-n} \\
\vdots \\
y_{k-1}
\end{array}\right],
$$

where $g^{T} \equiv\left(g_{n}, \cdots, g_{1}\right)$. Thus, the closed-loop system

$$
x_{k}=\left(A+b f^{T}\right) x_{k-1},
$$

goes over to the closed-loop system

$$
y_{k}+\left(a_{1}-g_{1}\right) y_{k-1}+\cdots+\left(a_{n}-g_{n}\right) y_{k-n}=0 .
$$


Let $\lambda_{1}, \cdots, \lambda_{\mathrm{n}}$ denote the (not necessarily distinct) eigenvalues of A. We are assuming that the entries in $A$ are all real; hence $\left\{\lambda_{1}, \cdots, \lambda_{n}\right\}$ is symmetric with respect to the real axis in the complex plane. Given any likewise symmetric set of complex numbers $\left\{\nu_{1}, \cdots, \nu_{n}\right\}$, it is now an easy matter to construct a feedback gain $f \varepsilon \mathbb{R}^{n}$ for which the eigenvalues of $A+b f^{T}$ are precisely $\left\{\nu_{1}, \cdots, \nu_{n}\right\}$ Indeed, if

$$
\lambda^{n}+c_{1} \lambda^{n-1}+\cdots+c_{n} \equiv \prod_{i=1}^{n}\left(\lambda-\nu_{i}\right),
$$

then $c_{i} \in \mathbb{R}$ for $i=1, \cdots, n$ and hence, referring to (1.10), we must have

$$
g_{i}=a_{1}-c_{i} \quad i=1, \cdots, n
$$

or

$$
\mathrm{g}^{\mathrm{T}}=\mathrm{a}^{\mathrm{T}}-\mathrm{c}^{\mathrm{T}} \quad \text { where } \mathrm{a}^{\mathrm{T}}=\left(\mathrm{a}_{\mathrm{n}}, \cdots, \mathrm{a}_{1}\right) \text {, etc }
$$

Referring to (1.9), we arrive at the Bass-Gura formula [10] for $f$ :

$$
f^{T}=(a-c)^{T} M^{-1} C^{-1}
$$

We will show that the same formula holds true under appropriate assumptions for the class of infinite dimensional systems mentioned above and to which we now turn. 
2. Nonharmonic Fourler Series and Controllability

In this section, we discuss the nature of the transformation which corresponds to the matrix $\mathrm{C}$ of the previous section. Before doing so, it is first necessary to recal1 some definitions which concern the scalar input system

$$
\dot{x}(t)=\mathscr{C l}_{x}(t)+b u(t)
$$

Let $\mathscr{A}$ generate a strong1y-continuous group $\{\mathscr{G}(t): t \varepsilon R\}$ on the Hilbert space $\mathscr{H}$, and denote the action of a conjugate linear functional $x$ on $\psi \varepsilon \mathscr{\&}$ by $\langle\psi, x\rangle$. As with the inner product $(\cdot, \cdot)$ on $\mathscr{H}$, the bracket $\langle\bullet, \bullet\rangle$ is understood to be conjugate linear in the first argument and linear in the second. It is also understood that $\langle\bullet, \bullet\rangle$ has the property that $\langle\psi, x\rangle=(\psi, x)$ if $x \in \mathscr{W}$. Let us define the weak solution $x(\cdot)$ to $(2.1)$ satisfying $x(0)=x_{0} \varepsilon \mathscr{H}$ by requiring

$$
\langle\psi, x(t)\rangle=\left\langle G(t)^{*} \psi, x_{0}\right\rangle+\int_{0}^{t}\left\langle G(t-s)^{*} \psi, b\right\rangle u(s) d s,
$$

for all $\psi \varepsilon \mathscr{D}\left(\mathscr{C}^{*}\right)$. Here, $\left\{\mathscr{G}(t)^{*}: t \in \mathbb{R}\right\}$ denotes the adjoint group generated by $\mathscr{Q}^{*}$ with domain $\left.\mathscr{D} \mathscr{C}^{*}\right) \subset \mathscr{H}$ and $b$ is understood to represent a conjugate linear functional on $\mathscr{\&}$.

\section{Definition 2.1. [4]:}

The control distribution element $b$ is said to be admissible with respect to $\mathscr{H}$ if $b$ can be identified with a conjugate linear functional, also denoted by $b$, for which

$$
\text { (i) } \mathscr{D}(\dot{b}) \geq \mathscr{D}\left(\mathscr{A}^{*}\right. \text {. }
$$


(ii) for any initial state $x(0) \varepsilon \mathbb{L}^{\prime}$ and any locally square integrable input $u$, the weak solution $x(t)$ of (2.1) lies in $\mathscr{H}$ for each $t>0$.

We note that if $\downarrow \varepsilon$ in $(2.1)$, then the weak solution coincides with the mild solution

$$
x(t)=\mathscr{G}(t) x_{0}+\int_{0}^{t} \mathscr{G}(t-s) b u(s) d s .
$$

Since $x(t) \varepsilon$ for each $t>0$ [5], $b$ in this case is admissible.

To make (2.2) somewhat more constructive, we introduce the sequence $\left\{\phi_{k}\right\}$ of eigenfunctions of $\mathscr{A}$ with corresponding eigenvalues $\left\{\omega_{k}\right\}$. We will assume that each $\omega_{k}$ is simple and that $\left\{\phi_{k}\right\}$ forms a Riesz basis for $\mathbb{Z}$ :

Definition 2.2 ([7], [11]):

A sequence $\left\{\phi_{k}\right\}$ of elements of a Hilbert space is said to form a Riesz basis for if the exist constants $d, D$ with $0<d<D<\infty$ such that every $\mathrm{x} \varepsilon \not$ may be expanded uniquely in a series

$$
\mathrm{x}=\sum_{\mathrm{k}} \mathrm{x}_{\mathrm{k}} \phi_{\mathrm{k}}
$$

with

$$
\mathrm{d}^{2} \sum_{\mathrm{k}}\left|\mathrm{x}_{\mathrm{k}}\right|^{2}<\| \mathrm{x}^{2} \not<\mathrm{D}^{2} \sum_{\mathrm{k}}\left|\mathrm{x}_{\mathrm{k}}\right|^{2}
$$

Equivalently ([7], e.g.), a Riesz basis may be thought of as the image of an orthonormal basis $\left\{e_{k}\right\}$ of $\Psi$ under a bounded and boundedly invertible transformation $\Phi$ :

$$
\phi_{\mathrm{k}}=\Phi \mathrm{e}_{\mathrm{k}}
$$


With every Riesz basis $\left\{\phi_{k}\right\}$, there is associated a unique "dual" Riesz basis $\left\{\psi_{k}\right\}$ defined by

$$
\psi_{k}=\left(\Phi^{*}\right)^{-1} e_{k}
$$

with the biorthonormality property $\left(\psi_{k}, \phi_{\ell}\right)_{\mathcal{H}}=\delta_{k \ell} \cdot \quad$ The expansion coefficients of $x \in \mathcal{H}$ with respect to $\left\{\phi_{k}\right\},\left\{\psi_{k}\right\}$ are $\left(\psi_{k}, x\right),\left(x, \phi_{k}\right)$ respectively.

Let us denote by $\left\{\psi_{\mathrm{k}}\right\}$ the sequence of elgenfunctions of $\mathscr{A}^{*}$; 1.e., $\mathscr{A}^{*} \psi_{k}=\bar{\omega}_{k} \psi_{k}$. Suitably normalized, $\left\{\psi_{k}\right\}$ is easily seen to be dual to $\left\{\phi_{k}\right\}$. Now let $\psi=\psi_{k}$, in $(2.2)$ and set $x_{k}(t)=\left\langle\psi_{k}, x(t)\right\rangle$ and $b_{k}=\left\langle\psi_{k}, b\right\rangle$. Then

$$
x_{k}(t)=e^{\omega_{k} t} x_{k}(0)+b_{k} \int_{0}^{t} e^{\omega_{k}(t-s)} u(s) d s,
$$

and hence, weak solutions may be represented as

$$
x(t)=\sum_{k} x_{k}(t) \phi_{k}
$$

with the $x_{k}^{\prime}$ s given by (2.5). If sup $\mid$ Re $\omega_{k} \mid<\infty$, admissibility reduces, via (2.4), to whether or not, for each $t \geqslant 0$, the sequence

$$
\left\{b_{k} \int_{0}^{t} e^{w_{k}(t-s)} u(s) d s\right\} \varepsilon \ell^{2}
$$

for every $u \in L^{2}(0, t)$.

In the course of reducing (2.1) to canonical form, we will carry out manipulations similar to those employed in the previous section and which involve the expression 


$$
\int_{0}^{T} \mathscr{G}(\mathrm{T}-\mathrm{s}) \mathrm{bu}(\mathrm{s}) \mathrm{ds}
$$

The whole point of the above discussion is that the latter expression may, even if $b \notin \mathcal{H}$, be interpreted rigorously if the eigenfunctions $\left\{\phi_{k}\right\}$ of form a Riesz basis for $\mathscr{H}$ and $b$ is admissible with respect to $\mathscr{A}$ :

$$
\int_{0}^{T} \mathscr{G}(t-s) b u(s) d s \equiv \sum_{k}\left(b_{k} \int_{0}^{t} e^{\mu_{k}(t-s)} u(s) d s\right) \phi_{k}
$$

In particular, if $x_{0}=0$, we may write

$$
\mathrm{x}(\mathrm{T})=\int_{0}^{\mathrm{T}} \mathscr{G}(\mathrm{T}-\mathrm{s}) \mathrm{bu}(\mathrm{s}) \mathrm{ds} \equiv \mathscr{C}(\mathrm{T}) \mathrm{u}
$$

This is the analogue of (1.4). Conversely, if $x \in \mathcal{H}$, the problem of constructing a control $u \in L^{2}(0, T)$ which takes the origin to $x$ at time $\mathrm{T}$ is characterized by. a moment problem:

$$
\mathscr{C}(\mathrm{T}) \mathrm{u}=\mathrm{x},
$$

$$
\begin{aligned}
& \text { or, with } x=\sum_{k} x_{k} \phi_{k}, \\
& \qquad b_{k} \int_{0}^{T} e^{\omega_{k}(T-s)} u(s) d s=x_{k} .
\end{aligned}
$$

Definition 2.3 ([15]):

The pair (\&, b) is said to be approximately, exactly controllable in

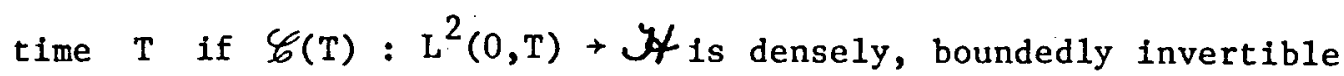
respectively. 
Referring to (2.7), it is obvious that approximate controllability requires $b_{k} \neq 0$ for all $k$. But controllability also depends upon properties of the sequence of exponentials $\left\{e^{\omega_{k}}\right\}$. This paper is concerned with a very special class of $\mathscr{A}^{\prime}$ s, namely those whose spectrum coincides with the zero set $\left\{\omega_{k}\right\}$ of a function having the form

$$
p(\omega)=e^{\omega T}+a_{1} e^{\omega\left(T-\theta_{1}\right)}+\cdots+a_{m}+\int_{0}^{T} a(\theta) e^{\omega(T-\theta)} d \theta
$$

It is assumed that $a_{1} \in \mathbb{R} i=1, \cdots, m$ with $a_{m} \neq 0$, that $a(\cdot) \in L^{2}(0, T)$ is real valued, and that

$$
0<\theta_{1}<\cdots<\theta_{\mathrm{m}} \equiv \mathrm{T} \text {. }
$$

Let us write

$$
p(\omega)=p_{0}(\omega)+\int_{0}^{T} a(\theta) e^{\omega(T-\theta)} d \theta
$$

and let $\left\{\sigma_{k}\right\}$ denote the zero set of the exponential polynomial $p_{0}$. It is easy to see that the set $\left\{\sigma_{k}\right\}$ as well as the set $\left\{y_{k}\right\}$ must lie in a vertical strip of finite width in the complex plane. It has been shown [1] using the argument principle that an infinite number of $\sigma_{k}$ 's do exist and that the number of $\sigma_{k}$ 's in any horizontal strip of fixed height is bounded.

The prototype in this situation is $p_{0}(\omega)=e^{2 \omega}-1$ which has for its set of zeros $\left\{\sigma_{k}\right\}=\{k \pi i\}$. It can also be shown [17], [19], using the above properties of $\left\{\sigma_{k}\right\}$ together with the Fourier transform in the complex domain, that the sequence of exponentials $\left\{e^{\sigma_{k}}\right\}$ forms a Riesz basis for $L^{2}(0, T)$ if, in addition, $\underset{k}{\inf }\left|\mathrm{p}_{\mathrm{o}}^{\prime}\left(\sigma_{\mathrm{k}}\right)\right|>0$. The latter condition ensures the bounded invertibility of the map $\Phi$ discussed after Definition 2.2. One can also demonstrate using Rouche's theorem that the sequence $\left\{a_{k}\right\}$ of zeros of $p$ may be indexed in such a way that $\left\{\omega_{k}-\sigma_{k}\right\} \varepsilon l^{2}$ and that 
inf $\left|p^{\prime}\left(\omega_{k}\right)\right|>0$ is sufficient to yield the Riesz basis property for the sequence $\left\{\mathrm{e}^{\omega_{k}}\right\}$. We omit the somewhat detailed proof of these statements [19] and return to their relationship with the notion of controllability. Let us denote the sequence biorthonormal to $\left\{e^{\omega_{k}}\right\}$ by $\left\{q_{\ell}(\cdot)\right\}$ :

$$
\int_{0}^{T} \bar{q}_{\ell}(s) e^{\omega_{k} s} d s=\delta_{k \ell} .
$$

The construction of the sequence $\left\{q_{\ell}(\cdot)\right\}$ goes back to the work of Paley and Wiener [12] and is carried out as follows. One forms the function

$$
\hat{q}(\omega)=\frac{p(\omega)}{p^{\prime}\left(\omega_{k}\right)\left(\omega-\omega_{k}\right)},
$$

and notes that $\hat{q}_{\ell}\left(w_{k}\right)=\delta_{k \ell}$. The Paley-Wiener theorem $([12],[13])$ then asserts that $\hat{\mathrm{q}}_{\ell}$ is the Laplace transform of a square integrable function $\bar{q}_{\ell}(\cdot)$ whose support $\operatorname{supp}\left(q_{\ell}\right) \subseteq[0, T]$ :

$$
\hat{q}_{\ell}(\omega)=\int_{0}^{T} e^{\omega s} \bar{q}_{\ell}(s) d s
$$

Thus, $\bar{q}_{\ell}$ may be represented via the inverse Laplace transform

$$
\bar{q}_{\ell}(s)=\frac{1}{2 \pi i} \int_{-i \infty}^{1 \infty} e^{-s \omega} \hat{q}_{\ell}(\omega) d \omega
$$

The sequence $\left\{q_{\ell}(\cdot)\right\}$ so constructed is biorthonormal to $\left\{e^{u k}\right\}$ since

$$
\int_{0}^{T} \bar{q}_{\ell}(s) e^{\omega_{k} s} d s=\hat{q}_{\ell}\left(\omega_{k}\right)=\delta_{k \ell} .
$$


However, in the next section, we shall derive an explicit formula for each $\mathrm{q}_{2}$ without any mention of the Paley-Wiener theory. In effect, the inverse transform (2.9) can be computed explicitly in closed form.

Assuming $b_{k} \neq 0$ for any $k$, we may now invert relationship (2.7) with the aid of the sequence $\left\{q_{k}(\cdot)\right\}$. Indeed, the control

$$
u(t)=\sum_{k} \frac{x_{k}}{b_{k}} \bar{q}_{k}(T-t),
$$

satisfies (2.7). Here, $T$ is understood to be the quantity appearing in the definition (2.8) of p. Thus $\mathscr{C} \equiv \mathscr{C}(\mathrm{T})$ has the properties

$$
\begin{array}{r}
\mathscr{C}: \overline{\mathrm{q}}_{\mathrm{k}}(\mathrm{T}-\bullet) \rightarrow \mathrm{b}_{\mathrm{k}} \phi_{\mathrm{k}} \\
\mathscr{C}^{-1}: \phi_{k} \rightarrow \mathrm{b}_{\mathrm{k}}^{-1} \overline{\mathrm{q}}_{\mathrm{k}}(\mathrm{T}-\cdot),
\end{array}
$$

which will be used throughout the remainder of the paper.

We conclude this section with a summary of the various assumptions made and a brief discussion of their significance.

\section{Assumption 2.4:}

(i) $\mathscr{A}$ generates a strongly-continuous group. $\{\mathscr{G}(t): t \varepsilon \mathbb{R}\}$ on a Hilbert space $\mathscr{H}$.

(11) The sequence of eigenfunctions $\left\{\phi_{k}\right\}$ of $\mathcal{A}$ forms a Riesz basis for $\sharp$.

(iii) The elgenvalues $\left\{\omega_{k}\right\}$ of $\mathscr{A}$ are the roots of $p(\omega)$ given by (2.8) and $p^{\prime}\left(\omega_{k}\right) \neq 0$ for any $k$.

(iv) The control distribution element $b$ is admissible with respect to with coefficients $b_{k}=\left\langle\psi_{k}, b\right\rangle \neq 0$ for any $k$. $\left.\psi_{k}^{*} \psi_{k}=\bar{c}_{k} \psi_{k}\right)$. 
Since finite 1inear combinations of the $\phi_{k}{ }^{\prime} s$ are dense in $\mathcal{H}_{\text {, the pair }}$ (Qd,b), under the above assumption, is approximately controllable in time $T$ (c.f. [15]). If $\inf \left|b_{k}\right|>0$ and inf $\left|p^{\prime}\left(\omega_{k}\right)\right|>0$, exact controllability in time. $T$ may be established as we11 [15].

Part (iii) of the above assumption essentially limits the scope of our results to the class of linear hyperbolic systems described in the introduction. For such systems, $p^{\prime}\left(\omega_{k}\right) \neq 0$ for all $k$ does not hold true in general. We make this hypothesis here only to simplify our presentation notwithstanding a good dose of tedium, it can be shown by introducing generalized exponentials (i.e., $t^{j} e^{\omega_{k} t}$ ) in the case of multiple roots $q_{k}$ that the canonical form presented in this paper remains valid.

The case of boundary control (see equation (7)), where $b \cdot \varepsilon \mathbf{R}^{n}$, does correspond to an admissible distribution $b$ ([15], Theorem 3.1)

$$
b_{k}=\left\langle\psi_{k}, b\right\rangle=\psi_{k}^{+}(1)^{*} \Lambda^{+}(1) b \text {, }
$$

and, if no component of $b$ vanishes, approximate conntrollability may be established [19]. We do not wish to emphasize the abstract character of the above assumptions. However, the development of a canonical form is carried out most transparently in this setting.

\section{Reduction to Canonical Form}

In this section, we will derive the transformation which carries the system (2) to its canonical form (3), and in order to pursue as closely as possible the analogy between the pair $(\mathrm{A}, \mathrm{b})$ of the discrete system above with the pair $(\mathscr{A}, b)$ at hand, we will proceed somewhat formally. Assumption 
2.4 will be understood to be in force throughout. We begin with an analogue of the Cayley-Hamflton theory:

Lemma 3.1: Under (i), (ii), and (iii) of Assumption 2.4,

$$
\mathscr{G}(t)+a_{1} \mathscr{G}\left(t-\theta_{1}\right)+\cdots+a_{m} \mathscr{G}(t-T)+\int_{0}^{T} a(\theta) \mathscr{G}(t-\theta) d \theta \equiv 0 .
$$

Proof. Given an arbitrary initial state $x=\sum_{k} x_{k} \phi_{k} \varepsilon_{\mathcal{H}} H_{\text {, the solution to }}$ $\dot{x}(t)=\mathscr{L} x(t)$ is given formally by $x(t)=\mathscr{G}(t) x$ and concretely by

Thus

$$
x(t)=\sum_{k} e^{\omega_{k} t} x_{k} \phi_{k}
$$

$$
\begin{array}{r}
{\left[\mathscr{G}(t)+a \mathscr{G}_{1}\left(t-\theta_{1}\right)+\cdots+a_{m} \mathscr{G}(t-T)+\int_{0}^{T} a(\theta) \mathscr{G}(t-\theta) \mathrm{d} \theta\right] \mathrm{x}} \\
=\sum_{k} p\left(\omega_{k}\right) e^{\omega_{k}(t-T)} x_{k} \phi_{k}=0 .
\end{array}
$$

This completes the proof of the lemma.

$$
\begin{aligned}
& \text { Formally, solutions to } \dot{x}(t)=\mathscr{A x}(t)+b u(t) \text { satisfy } \\
& \qquad x(t+r)=\mathscr{G}(r) x(t)+\int_{0}^{r} \mathscr{g}(r-s) b u(s) d s .
\end{aligned}
$$

Thus, 


$$
\begin{aligned}
& x(t+T)+a_{1} x\left(t+T-\theta_{1}\right)+\cdots+a_{m} x(t)+\int_{0}^{T} a(\theta) x(t+T-\theta) d \theta \\
& =\left[\mathscr{G}(t)+a_{1} \mathscr{G}\left(t-\theta_{1}\right)+\cdots+a_{m} \mathscr{G}(t-T)+\int_{0}^{T} a(\theta) \mathscr{G}(t-\theta) d \theta\right] x(T) \\
& +\int_{0}^{T} \mathscr{G}(T-s) b u(t+s) d s+a_{1} \int_{0}^{T-\theta_{1}} \mathscr{G}\left(T-\theta_{1}-s\right) b u(t+s) d s \\
& +\cdots+a_{m-1} \int_{0}^{T-\theta}{ }_{m-1}\left(T-\theta_{m-1}-s\right) b u(t+s) d s \\
& +\int_{0}^{T} a(\theta) \int_{0}^{T-\theta} \mathscr{G}(T-\theta-s) b u(t+s) d s d \theta .
\end{aligned}
$$

The first term is zero by Lemma 3.1. Changing the inner variable of integration from $s$ to $s+\theta$ and then interchanging the order of integration, the last term may be written as

$$
\int_{0}^{\mathrm{T}} \mathscr{G}(\mathrm{T}-\mathrm{s}) b \int_{0}^{\mathrm{s}} \mathrm{a}(\theta) \mathrm{u}(\mathrm{t}+\mathrm{s}-\theta) \mathrm{d} \theta \mathrm{ds}
$$

Likewise,

$$
\begin{aligned}
\int_{0}^{\mathrm{T}-\theta_{1}} \mathscr{G}\left(\mathrm{T}-\theta_{i}-\mathrm{s}\right) \mathrm{bu}(\mathrm{t}+\mathrm{s}) \mathrm{d} s & =\int_{0}^{\mathrm{T}} \mathscr{G}\left(\mathrm{T}-\theta_{i}-\mathrm{s}\right) b_{\mathrm{X}_{\left[0, T-\theta_{i}\right]}(\mathrm{s}) \mathrm{u}(\mathrm{t}+\mathrm{s}) \mathrm{ds}} \\
& =\int_{0}^{\mathrm{T}} \mathscr{G}(\mathrm{T}-\mathrm{s}) b \mathrm{x}_{\left[0, \mathrm{~T}-\theta_{i}\right]}\left(\mathrm{s}-\theta_{i}\right) \mathrm{u}\left(\mathrm{t}+\mathrm{s}-\theta_{i}\right) \mathrm{ds} \\
& =\int_{0}^{\mathrm{T}} \mathscr{G}(\mathrm{T}-\mathrm{s}) b \mathrm{x}_{\left[\theta_{i}, \mathrm{~T}\right]}(\mathrm{s}) \mathrm{u}\left(\mathrm{t}+\mathrm{s}-\theta_{i}\right) \mathrm{ds},
\end{aligned}
$$

where $x_{[a, b]}(\cdot)$ is the characteristic function of the interval $[a, b]$. The right-hand side of (3.2) may now be written as 


$$
\int_{0}^{\mathrm{T}} \mathscr{G}(\mathrm{T}-\mathrm{s}) b(\mathscr{H u}(\mathrm{t}+\cdot))(\mathrm{s}) \mathrm{ds} \equiv \mathscr{C H} \mathrm{u}(\mathrm{t}+\cdot \cdot)
$$

where $\mathscr{C}$ is as before and $\mathscr{H}: \mathrm{L}^{2}(0, \mathrm{~T})+\mathrm{L}^{2}(0, \mathrm{~T})$ is the transformation defined by

$$
\begin{aligned}
(\mathscr{H} y)(s)=y(s) & +a_{1} x_{\left[\theta_{1}, T\right]}(s) y\left(s-\theta_{1}\right)+\cdots \\
& +a_{m-1} x_{\left[\theta_{m-1}, T\right]}(s) y\left(s-\theta_{m-1}\right)+\int_{0}^{s} a(\theta) y(s-\theta) d \theta .
\end{aligned}
$$

Equation (3.2) now reads

$$
x(t+T)+a_{1} x\left(t+T-\theta_{1}\right)+\cdots+a_{m} x(t)+\int_{0}^{T} a(\theta) x(t+T-\theta) d \theta=\mathscr{C H} u(t+\cdot)
$$

Equations (3.3) and (3.4) should be compared with (1.6) and (1.7) respectively. To proceed further, the nature of the transformation $\mathscr{H}$ must be analyzed.

Lemma 3.2:

(1) $\mathscr{M}$ is bounded and boundedly invertible.

(ii) If $g \varepsilon L^{2}(0, T)$ with $\operatorname{supp}(g) \subset[T-\theta, T]$ for some $\theta \varepsilon(0, T)$, then $\operatorname{supp}\left(K^{-1} \mathrm{~g}\right) \subset[\mathrm{T}-\theta, \mathrm{T}]$ as wel1.

Proof: That $\mathscr{H}$ is bounded is clear from (3.3). Referring to the latter, let us write

$$
\mathscr{M}=\mathscr{P}-\mathscr{N}_{1}-\mathscr{N}_{2}
$$

with $\left(\mathscr{N}_{2} \mathrm{y}\right)(\mathrm{s}) \equiv-\int_{0}^{s} \mathrm{a}(\theta) \mathrm{y}(\mathrm{s}-\theta) \mathrm{d} \theta$. A standard theorem from integral 
equations [8] yields the fact that $\left(\mathscr{\Psi}-\mathscr{N}_{2}\right)^{-1}$ exists and is bounded with

$$
\left(\mathscr{P}=\mathscr{N}_{2}\right)^{-1}=\mathscr{P}+\sum_{\mathrm{k}=1}^{\infty} \mathscr{N}_{2}^{\mathrm{k}} \text {. }
$$

It is easily established that $\operatorname{supp}\left(\mathscr{N}_{2} \mathrm{~g}\right)$ and hence $\left(\left(\mathcal{T}-\mathscr{N}_{2}\right)^{-1} \mathrm{~g}\right) \subseteq[\mathrm{T}-\theta, \mathrm{T}]$

if $\operatorname{supp}(\mathrm{g}) \subseteq[\mathrm{T}-\theta, \mathrm{T}]$. Moreover, $\mathscr{N}_{1}$ is nilpotent since

$\operatorname{supp}(\mathscr{N}, g) \subseteq\left[\mathrm{T}-\theta+\theta_{1}, \mathrm{~T}\right]$ if $\operatorname{supp}(g) \subseteq[\mathrm{T}-\theta, \mathrm{T}]$. Thus $\left(\mathcal{F}-\mathscr{N}_{2}\right)^{-1} \mathscr{N}_{1}$ is

nilpotent and

$$
\begin{aligned}
\mathscr{M}^{-1} & =\left(\mathscr{F}-\mathscr{N}_{2}\right)^{-1}\left(\mathscr{F}-\left(\mathscr{P}-\mathscr{N}_{2}\right)^{-1} \mathscr{N}_{1}\right)^{-1} \\
& \left.=\left(\mathscr{F}-\mathscr{N}_{2}\right)^{-1}\left(\mathscr{P}+\sum_{\mathrm{k}=1}^{\mathrm{n}}\left(\mathscr{P}-\mathscr{N}_{2}\right)^{-1} \mathscr{N}_{1}\right)^{\mathrm{k}}\right),
\end{aligned}
$$

for some $\mathrm{n}<\infty$, which shows that $\mathscr{M}^{-1}$ is bounded and satisfies (ii). This completes the proof.

We remark that part (ii) of Lemma 3.2 is the analogue of the fact that $\operatorname{span}\left\{e_{n-p}, \cdots, e_{n}\right\}$ is invariant under the matrix $M^{-1}$ discussed in Section 1. We next Fourier analyze the action of $\mathscr{H}$ on the exponentials $e^{u_{k}^{*}}$ :

Lemma 3.3:

$$
\int_{0}^{T} e^{\omega(t-s)}\left(\mathscr{k} e^{\omega_{k}}\right)(s) d s=p(\omega) /\left(\omega-\omega_{k}\right) .
$$

Proof: In forming the expression on the left, we find terms of the form

$$
\int_{0}^{T} e^{\omega(T-s)} \chi_{\left[\theta_{i}, T\right]}(s) e^{\omega_{k}\left(s-\theta_{i}\right)} d s,
$$

and the term

$$
\int_{0}^{T} e^{\omega_{k}(T-s)} \int_{0}^{s} a(\theta) e^{\mu_{k}(s-\theta)} d \theta d s .
$$


Standard manipulations reduce the former to

$$
\frac{e^{\omega\left(T-\theta_{i}\right)}-e^{\omega_{k}\left(T-\theta_{i}\right)}}{\omega-\omega_{k}},
$$

and the latter to

$$
\int_{0}^{T} a(\theta) \frac{e^{\omega(T-\theta)}-e^{\omega_{k}(T-\theta)}}{\omega-\omega_{k}} d \theta .
$$

It follows that

$$
\begin{gathered}
\int_{0}^{T} e^{\omega(T-s)}\left(H e^{\omega_{k}}\right)(s) d s=\left[e^{\omega T}-e^{\omega_{k} T}+a_{1}\left(e^{\omega\left(T-\theta_{1}\right)}-e^{\omega_{k}\left(T-\theta_{1}\right)}\right)\right. \\
+\cdots+a_{m-1}\left(e^{\omega\left(T-\theta_{m-1}\right)}-e^{\omega_{k}\left(T-\theta_{m-1}\right)}\right) \\
\left.+\int_{0}^{T} a(\theta)\left(e^{\omega(T-\theta)}-e^{\omega_{k}(T-\theta)}\right) d \theta\right] /\left(\omega-\omega_{k}\right) \\
=\left(p(\omega)-p\left(\omega_{k}\right)\right) /\left(\omega-\omega_{k}\right)=p(\omega) /\left(\omega-\omega_{k}\right),
\end{gathered}
$$

since $\omega_{k}$ is a zero of $p$. This completes the proof.

Recal1 that the basis $\left\{e^{\omega_{k}}\right\} \subset L^{2}(0, T)$ has a unique biorthonormal sequence which we denote by $\left\{\mathrm{q}_{\mathrm{k}}(\cdot)\right\}<\mathrm{L}^{2}(0, \mathrm{~T})$. The key property which $\mathscr{H}$ possesses is the following:

Lemma 3.4:

$$
\mathscr{H}: \mathrm{e}^{\omega_{k} \cdot}+\mathrm{p}^{\prime}\left(\omega_{\mathrm{k}}\right) \bar{q}_{\mathrm{k}}(\mathrm{T}-\cdot)
$$


Proof: According to Lemma 3.3 ,

$$
\int_{0}^{T} e^{\omega_{j}(T-s)}\left(\mathbb{K}^{\omega_{k}}\right)(s) d s=p^{\prime}\left(\omega_{k}\right) \delta_{j k} \cdot
$$

Since the sequence $\left\{q_{j}(T-\cdot)\right\}$ is biorthonormal to $\left\{e^{\omega_{j}(T-\bullet)}\right\}$ and unique, we conclude that

$$
\left(\mathscr{H} e^{\omega_{k} \cdot}\right)(s)=p^{\prime}\left(\omega_{k}\right) \bar{q}_{k}(T-s),
$$

which completes the proof.

\section{Remark:}

The preceding lemma allows us to construct the biorthonormal sequence explicitly:

$$
\bar{q}_{k}(s)=\frac{1}{p^{\prime}\left(\omega_{k}\right)}\left(K e^{\omega_{k}}\right)(T-s)
$$

Under Assumption 2.4, (iv), the map $\mathscr{C}$ possesses a densely defined inverse $\mathscr{C}^{-1}($ see $(2.11))$. If $x(\cdot)$ is a solution of $\dot{x}=\mathscr{A x}+$ bu with sufficiently regular initial state $x_{0}$ and input $u(\cdot)$, we may define

$$
n(t, \cdot)=\left(\mathscr{H}^{-1} \mathscr{C}^{-1} x(t+T)\right)(\cdot) \varepsilon L^{2}(0, T)
$$

Let us assume that $n$ is defined for every $t$ and $s$. Then

$$
\text { Lemma 3.5: } \quad n(t, s) \text { is a function of } s+t \text {. }
$$

Proof: Suppose $p+s=r<T$. We wish to show that $n(t, r)=n(t+p, s)$. According to (3.5), 


$$
n(t, r)=\left(\mathscr{C}^{-1} \mathscr{C}^{-1} x(t+T)\right)(r) .
$$

If we let $x(t+T)=\sum_{k} x_{k}(t+T) \phi_{k}$ and use (2.11) and Lemma 3.4, we have

$$
n(t, r)=\sum_{k} p^{\prime}\left(\omega_{k}\right)^{-1} b_{k}^{-1} x_{k}(t+T) e^{\omega_{k} r}
$$

Likewise,

$$
\eta(t+p, s)=\sum_{k} p^{\prime}\left(\omega_{k}\right)^{-1} b_{k}^{-1} x_{k}(t+p+T) e^{\omega_{k} s} \text {. }
$$

Since

$$
x_{k}(t+p+T)=e^{\omega_{k} p} x_{k}(t+T)+b_{k} \int_{0}^{p} e^{w_{k}(p-\theta)} u(t+\theta+T) d \theta
$$

we have

$$
n(t+p, s)-n(t, r)=\sum_{k}\left(p^{\prime}\left(\omega_{k}\right)^{-1} \int_{0}^{p} e^{\omega_{k}(p-\theta)} u(t+\theta+T) d \theta\right) e^{\omega_{k} s} .
$$

If we change the variable of integration via $p-\theta=T-\tau$ and invoke Lemma 3.4, the right-hand side becomes

$$
=\left(K^{-1} \sum_{k} \int_{T-p}^{T} e^{\omega_{k}(T-\tau)} u(t+p+\tau) d \tau \bar{q}_{k}(T-\cdot)\right)(s) .
$$

But the function on which $\mathscr{M}^{-1}$ operates is nothing but $\mathrm{X}_{[\mathrm{T}-\mathrm{p}, \mathrm{T}]}(\cdot) \mathrm{u}(\mathrm{t}+\mathrm{p}+\bullet)$ expanded in terms of the sequence $\left\{\bar{q}_{k}(T-\bullet)\right\}$. Hence

$$
\begin{aligned}
& =\left(\mathbb{K}^{-1} x_{[T-p, T]}(\cdot) u(t+p+\cdot)\right)(s) \\
& \left.\equiv \mathscr{K}^{-1} g\right)(s) .
\end{aligned}
$$

Clearly, $\operatorname{supp}(\mathrm{g})<[\mathrm{T}-\mathrm{p}, \mathrm{T}]$, and since $0<\mathrm{p}+\mathrm{s}=\mathrm{r}<\mathrm{T}$ implies $\mathrm{s} \varepsilon[0, \mathrm{~T}-\mathrm{p}]$, 
we conclude from Lemma 3.2 that $\left.S_{\mathscr{C}^{-1}} g\right)(s)=0$. Thus, $n(t+p, s)=n(t, r)$. This completes the proof.

Lemma 3.5 may be shown to hold true in the case where $n(t, \cdot)$ is merely a square-integrable function for each $t$ by approximating everything with continuous functions and then passing to the limit. We may now define the scalar varlable

$$
y(t+\cdot)=n(t, \cdot)=\left(\mathscr{M}^{-1} \mathscr{C}^{-1} x(t+T)\right)(\cdot)
$$

We conclude this section with

Theorem 3.6: Under Assumption (2.4), the variable y defined by (3.6) satisfies the functional equation

$$
y(t)+a_{1} y\left(t-\theta_{1}\right)+\cdots+a_{m} y(t-T)+\int_{0}^{T} a(\theta) y(t-\theta) d \theta=u(t)
$$

Proof: $\quad$ Apply $\mathscr{K}^{-1} \mathscr{C}^{-1}$ on the left to both sides of (3.4).

\section{Spectral Synthesis}

Let us first examine how continuous state feedback

$$
u(t)=(f, x(t)
$$

In the original system (2.1) manifests 1 tself. in the canonical system (3.7). Let .6. have the expansion 


$$
f=\sum_{j} \mathrm{f}_{j} \psi_{j}
$$

where $\left\{\psi_{j}\right\}$ denotes the sequence of eigenfunctions of $\mathscr{A}^{*}$. Let $x(t)$ and $y(t+\bullet)$ have the expansions

$$
\begin{aligned}
x(t) & =\sum_{k} x_{k}(t) \phi_{k} \\
y(t+\bullet) & =\sum_{k} y_{k}(t) e^{\omega_{k} \cdot} .
\end{aligned}
$$

Referring to (3.6), we have

$$
x_{k}(t+T)=b_{k} p^{\prime}\left(\omega_{k}\right) y_{k}(t)
$$

Thus,

$$
u(t)=(6, x(t))_{\mathcal{L}}=\sum_{k} f_{k} b_{k} p^{\prime}\left(w_{k}\right) y_{k}(t-T)
$$

Again denoting the sequence biorthonormal to $\left\{e^{\omega_{k}}\right\}$ by $\left\{q_{j}(\cdot)\right\}$, the righthand side of the last equation may be written as

$$
\int_{0}^{T}\left(\sum_{j} f_{j} b_{j} p^{\prime}\left(w_{j}\right) \bar{q}_{j}(s)\right)\left(\sum_{j} y_{k}(t-T) e^{\omega_{k} s}\right) d s .
$$

By $(4.1)$,

$$
\sum_{k} y_{k}(t-T) e^{\omega_{k} s}=y(t-T+s)
$$

If we change the variable of integration via $s=T-\theta$ and define

$$
g(\theta) \equiv \sum_{j} f_{j} b_{j} p^{\prime}\left(\omega_{j}\right) \bar{q}_{j}(T-\theta),
$$


we obtain

$$
u(t)=\int_{0}^{T} g(\theta) y(t-\theta) d \theta
$$

The resulting closed-1oop canonical system is simply

$$
y(t)+a_{1} y\left(t-\theta_{1}\right)+\cdots+a_{m} y(t-T)+\int_{0}^{T}(a(\theta)-g(\theta)) y(t-\theta) d \theta=0 .
$$

Thus, the elgenvalues of the closed-loop system

$$
x(t)=(\mathscr{A}+b \otimes 6) x(t)
$$

are roots of

$$
p_{g}(\omega)=p_{0}(\omega)+\int_{0}^{T}(a(\theta)-g(\theta)) e^{\omega(T-\theta)} d \theta
$$

As we have already mentioned in Section 2, one can show using Rouche's theorem that the sequence $\left\{v_{k}\right\}$ of roots of $\mathrm{p}_{\mathrm{g}}$ differs from the sequence $\left\{\sigma_{k}\right\}$ of roots of $p_{0}$ in the sense that $\left\{v_{k}-\sigma_{k}\right\} \varepsilon \ell^{2}$. This suggests that any sequence $\left\{v_{k}\right\}$ with this property can be synthesized with a certain feedback gain $6 \varepsilon \mathbb{A}$.

Lemma 4.1: If $\left\{v_{k}\right\}$ is a sequence of distinct complex numbers for which $\left\{\nu_{k}-\sigma_{k}\right\} \varepsilon \ell^{2}$, then there exists a unique $c(\cdot) \varepsilon L^{2}(0, T)$ for which the zero set of

$$
p_{c}(\omega) \equiv p_{0}(\omega)+\int_{0}^{T} c(\theta) e^{\omega(T-\theta)} d \theta
$$

coincides with $\left\{v_{k}\right\}$. 
Proof: c is characterized by the moment problem

$$
p_{c}\left(\nu_{k}\right)=0 \Rightarrow \int_{0}^{T} c(\theta) e^{\nu_{k}(T-\theta)} d \theta=-p_{0}\left(\nu_{k}\right)
$$

Using the properties of the exponential polynomial $\mathrm{p}_{0}$ discussed in Section 2 together with the assumption that $\left\{v_{k}-\sigma_{k}\right\} \varepsilon \ell^{2}$, it is readily estab1ished that $\left\{p_{o}\left(\nu_{k}\right)\right\} \in \ell^{2}$. Moreover, it has been shown [17] that the sequence $\left\{\mathrm{e}^{\left.\nu_{\mathrm{k}}{ }^{\cdot}\right\}}\right.$ forms a basis for $\mathrm{L}^{2}(\mathrm{O}, \mathrm{T})$. Denoting the sequence biorthonormal to $\left\{e^{\nu_{k}}\right\}$ by $\left\{h_{k}(\cdot)\right\}$, the unique solution to the above moment problem is given by

$$
c(\cdot)=-\sum_{k} p_{0}\left(\nu_{k}\right) \bar{h}_{k}(T-\cdot),
$$

which completes the proof.

We now come to our main theorem.

Theorem 4.2: Let $\left\{v_{k}\right\}$ be àny sequence of distinct complex numbers for which

$$
\left\{\frac{\nu_{k}-\omega_{k}}{p^{\prime}\left(\omega_{k}\right) b_{k}}\right\} e^{2}
$$

and let Assumption 2.4 hold true. Then there exists a unique $6 \varepsilon$ f for which the spectrum of $\mathscr{A}+b \otimes 6$ coincides with $\left\{y_{k}\right\}$.

Proof. By Lemma 4.1, we may express the desired closed-loop characteristic function uniquely as

$$
p_{c}(\omega)=p_{0}(\omega)+\int_{0}^{T} c(\theta) e^{\omega(T-\theta)} d \theta \text {. }
$$

Referring to (4.2) and (4.4), the desired feedback gain element 
30

$$
\sigma=\sum_{k} f_{k} \psi_{k}
$$

must be such that

$$
p_{g}(\omega) \equiv p_{o}(\omega)+\int_{0}^{T}(a(\theta)-g(\theta)) e^{\omega(T-\theta)} d \theta=p_{c}(\omega),
$$

or $g=a-c$. Let $a$ and $c$ have the expansions

$$
\begin{aligned}
& a(\cdot)=\sum_{k} a_{k} \bar{q}_{k}(T-\cdot) \\
& c(\cdot)=\sum_{k} c_{k} \bar{q}_{k}(T-\cdot) .
\end{aligned}
$$

Referring to ( 4.2$)$, we must have

$$
f_{k} b_{k} p^{\prime}\left(w_{k}\right)=a_{k}-c_{k}
$$

or

$$
f_{k}=\left(a_{k}-c_{k}\right) p^{\prime}\left(\omega_{k}\right)^{-1} b_{k}^{-1}
$$

This should be compared with (1.11). It remains only to show that our hypotheses ensure that $\left\{f_{k}\right\} \varepsilon \ell^{2}$. The expansion coefficients $a_{k}$ and $c_{k}$ are given by

$$
\begin{aligned}
& a_{k}=\int_{0}^{T} a(\theta) e^{\omega_{k}(T-\theta)} d \theta=p\left(\omega_{k}\right)-p_{o}\left(\omega_{k}\right)=-p_{o}\left(\omega_{k}\right) \\
& c_{k}=\int_{0}^{T} c(\theta) e^{\omega_{k}(T-\theta)} d \theta=p_{c}\left(\omega_{k}\right)-p_{o}\left(\omega_{k}\right) .
\end{aligned}
$$


Thus, by the intermediate value theorem,

$$
a_{k}-c_{k}=-p_{c}\left(\omega_{k}\right)=-p_{c}\left(\nu_{k}\right)-p_{c}^{\prime}\left(\nu_{k}\right)\left(\omega_{k}-\nu_{k}\right)=p_{c}^{\prime}\left(\hat{\nu}_{k}\right)\left(\nu_{k}-\omega_{k}\right),
$$

for some $\hat{v}_{k}$ on the line segment connecting $v_{k}$ and $u_{k}$. Since $\sup _{k}\left|p_{c}^{\prime}\left(\hat{v}_{k}\right)\right|<\infty$, we conclude that

$$
\left\{\frac{a_{k}-c_{k}}{p^{\prime}\left(\omega_{k}\right) b_{k}}\right\} \in \ell^{2} \quad \text { just in case }\left\{\frac{v_{k}-\omega_{k}}{p^{\prime}\left(\omega_{k}\right) b_{k}}\right\} \varepsilon \ell^{2} \text {. }
$$

This completes the proof.

For linear hyperbolic systems of the type described in the introduction, Q7. is the real operator

$$
\mathscr{A}=\Lambda(\mathrm{x}) \frac{\mathrm{d}}{\mathrm{dx}}+\mathrm{A}(\mathrm{x}),
$$

with domain

$$
\mathscr{D}(\mathscr{D})=\left\{\phi \varepsilon \mathrm{H}_{2 \mathrm{n}}^{1}[0,1]: \phi^{-}(0)-\mathrm{D}_{0} \phi^{+}(0)=0=\phi^{+}(1)-\mathrm{D}_{1} \phi^{-}(1)\right\}
$$

where $H_{2 n}^{1}$ is the Sobolev space of square integrable $\phi^{2 n}$-valued functions possessing a square integrable derivative. As such the elgenfunctions of as well as those of $\mathscr{A}^{*}$ will have the property that $\bar{\phi}_{k}=\phi_{-k}$. Likewise, if $\left\{v_{k}\right\}$ is a sequence of complex numbers with $\bar{\nu}_{k}=\nu_{-k}$, then any biorthogonal set of functions $\left\{h_{k}(\cdot)\right\}$ associated with the exponentials $\left\{e^{{ }^{\nu}{ }^{\bullet}}\right\}$ will have the property that $\bar{h}_{k}=h_{-k}$. Thus, for any such sequence $\left\{v_{k}\right\}$ satisfying the hypothesis of Lemma 4.1 , the function $c(\cdot)$ will be real-valued with expansion coefficients satisfying $\bar{c}_{k}=c_{-k}$, and hence the feedback gain element 


$$
f=\sum_{k} f_{k} \psi_{k}=\sum_{k} \frac{a_{k}-c_{k}}{p^{\prime}\left(\omega_{k}\right) b_{k}} \psi_{k},
$$

constructed above w11l have each of its $2 n$ component functions real valued. We conclude this section with a brief discussion of how to use the above theory to construct a feedback gain which shifts a finfte number of elgenvalues. Suppose we wish to shift $\omega_{1}, \omega_{2}, \cdots, \omega_{n}$ to $\nu_{1}, \cdots, v_{n}$. The desired closed-loop characteristic function is thus

$$
p_{c}(\omega)=\frac{\alpha(\omega)}{\beta(\omega)} p(\omega)
$$

where $\alpha(\omega)=\prod_{k=1}^{n}\left(\omega-\nu_{k}\right)$ and $\beta(\omega)=\prod_{k=1}^{n}\left(\omega-\omega_{k}\right)$. Thus

$$
p_{c}(\omega)=p(\omega)+\frac{\alpha(\omega)-\beta(\omega)}{\beta(\omega)} p(\omega)
$$

Since the degree of $\alpha-\beta$ is strictly less than $n,(\alpha-\beta) / \beta$ admits a partial fractions decomposition

$$
\frac{\alpha(\omega)-\beta(\omega)}{\beta(\omega)}=\sum_{k=1}^{n} \frac{r_{k}}{\omega-\omega_{k}} .
$$

According to Lemma 3.3 ,

$$
\frac{p(\omega)}{\omega-\omega_{k}}=\int_{0}^{T}\left(K e^{\omega} k^{\cdot}\right)(\theta) e^{\omega(T-\theta)} d \theta
$$

Thus

$$
\mathrm{p}_{c}(\omega)=\mathrm{p}(\omega)+\int_{0}^{\mathrm{T}}\left(\mathscr{M} \sum_{\mathrm{k}=1}^{\mathrm{n}} \mathrm{r}_{k} \mathrm{e}^{\omega_{\mathrm{k}}}\right)(\theta) \mathrm{e}^{\omega(\mathrm{T}-\theta)} \mathrm{d} \theta \text {. }
$$

By Lemma 3.4, this is 


$$
\begin{aligned}
p_{c}(\omega) & =p(\omega)+\int_{0}^{T} \sum_{k=1}^{n} r_{k} p^{\prime}\left(\omega_{k}\right) \bar{q}_{k}(T-\theta) e^{\omega(T-\theta)} d \theta \\
& =p(\omega)+\int_{0}^{T} c(\theta) e^{\omega(T-\theta)} d \theta .
\end{aligned}
$$

Thus, the desired expansion coefficients of $c$ are given simply by $c_{k}=r_{k} p^{\prime}\left(\omega_{k}\right)$, with the residues $r_{k}$ being determined by $(4.6)$.

\section{Concluding Remarks}

In this paper, we have demonstrated precisely to what extent the efgenvalues associated with a controllable pair $(\mathcal{A}, b)$ of a certain type may be modified via continous linear state feedback. Our results parallel those of Russell [14] for the case $m=1$ as explained in the introduction. Our main contributions here include a more detalled analysis of the canonical transformation and a direct method for computing feedback gains in the case of shifting a finite number of eigenvalues. All of this requires, of course, a rather detailed knowledge of the spectral structure of $\mathcal{S}$. In practice, one can only obtain this information approximately by numerical computation. In this regard, our results might prove useful in providing exact solutions in special cases which could then be used to determine the accuracy of numerical computations in more general cases. It should be pointed out that it is unrealistic to assume the avallability of state feeback in systems of the type discussed in this paper. Nevertheless, we feel that the results herein obtained should play a role in the development of an observer or asymptotic state estimator theory for such systems. 


\section{REFERENCES}

[1] R. BELLMAN and K. COOKE, Different1a1-Difference Equations, Academic Press, New York, 1963.

[2] B.M. C. CLARKE and D. WILLIAMSON, "Control Canonical Forms and Efgenvalue Assignment by Feedback for a Class of LInear Hyperbolic Systems," SIAM J. Control Optim., 19, No. 6 (1981), pp. 711-729.

[3] R. COURANT and D. HILBERT, Methods of Mathematical Physics, Vol. II, Intersclence, 1962 .

[4] R. F. CURTAIN and A. J. PRITCHARD, "An Abstract Theory for Unbounded Control Action for Distributed Parameter Systems," SIAM J. Control Optim., 15 (1977), pp. 566-611.

[5] R..F. CURTAIN and A. J. PRITCHARD, Functional Analysis in Modern Applled Mathematics, Academic Press, London, 1977, p. 159.

[6] S. FRANKEL, Multiconductor Transmission L1ne Analysis, Artech House, 1977.

[7] J. HIGGINS, Completeness and Basts Properties of Sets of Spectal Functlons, Cambridge UnIversity Press, New York, 1977.

[8] H. HOCHSTADT, Integral Equations, Wlley-Intersclence, New York, 1973, p 33. 
[9] IFAC Symposium on the Control and Distributed Parameter Systems, June 1971 Conference Proceedings, Banff, Canada, papers 6-1, 6-2, and 6-3.

[10] T. KAILATH, Linear Systems, Prentice-Ha11, NY, 1980, pp. 199-201.

[11] M. G. KREIN AND I. C. GOHBERG, Introduction to the Theory of Nonselfadfoint Operators, Translations of Mathematical Monographs, Vol. 18, Amer. Math. Soc., Providence, RI, 1969.

[12] R. E. A. C. PALEY and N. WIENER, Fourier Transforms in the Complex Domain, Amer. Math. Soc. Colloquiuum Publications 19, 1934.

[13] W. RUDIN, Real and Complex Analysis, McGraw Hi11, New York, 1966, Chapter 19.

[14] D. L. RUSSELL, "Canonical Forms and Spectral Determination for a Class of Hyperbolic Distributed Parameter Control Systems," J. Math. Ana1. 62, No. 1 (1978), pp. 186-225.

[15] D. L. RUSSELL, "Controllability and Stablizability Theory for Linear Partial Differential Equations: Recent Progress and Open Questions," SIAM Rev., 20, No. 4 (1978), pp. 639-739.

[16] D. L. RUSSELL, Mathematics of Finite-Dimenslonal Control Systems, Marce1 Dekker, New York, 1979, p. 126. 
[17] D. L. RUSSELL, "Nonharmonic Fourier Series in the Control Theory of Distrfbuted Parameter Systems," J. Math. Anal. App1., 18, (1967), pp. 542-559.

[18] S.-H. SUN, "On Spectrum Distribution of Completely Controllable Linear Systems, SIAM J. Contro1 Optim., 19, No. 6 (1981), pp. 730-744.

[19] R. TEGLAS, "A Contro1 Canonical Form for a Class of Linear Hyperbolic Systems," Doctoral Thesis, Mathematics Department, University of Wisconsin-Madison, 1981 . 


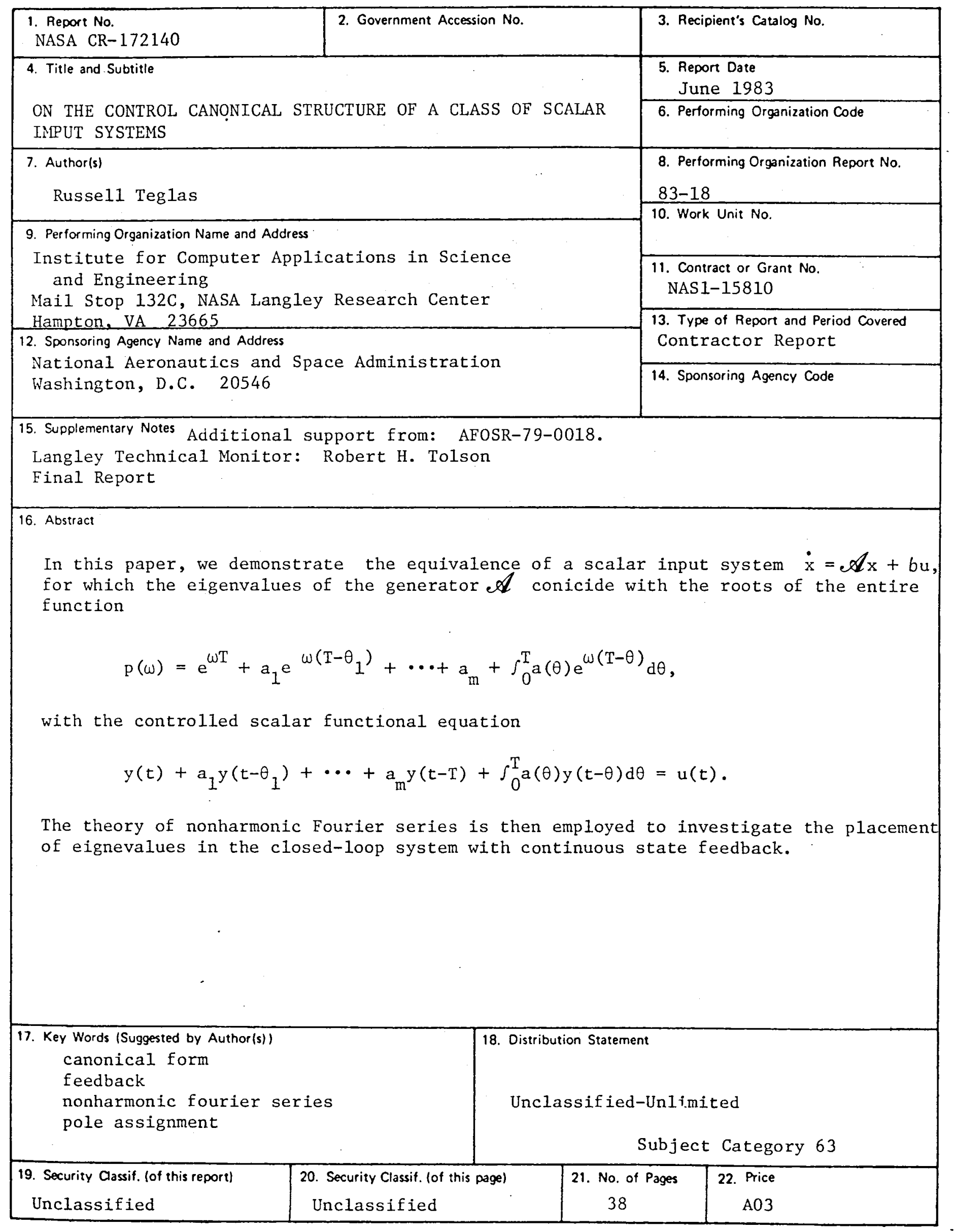


End of Document 